\title{
¿Y si investigamos, Señor Poe?
}

Federico PATÁN

Universidad Nacional Autónoma de México

\begin{abstract}
Poe es considerado uno de los iniciadores de la literatura policiaca. Partiendo de esa premisa, el presente ensayo examina, en cuatro de sus cuentos, aquellos elementos que dan soporte a la idea de arranque: la creación del investigador privado, la creación de un personaje que se encarga de la narración y el establecimiento de una variante de lo policiaco que da pie al desarrollo de la literatura de enigma.

Por tanto, se hacen comparaciones con la otra tendencia, la establecida hacia los años veintes por autores estadounidenses como Hammett y Chandler.
\end{abstract}

PALABRAS CLAVE: crimen, detective, investigación, narrador.

It is a common feature to give Poe the paternity of detective fiction. The following pages explore that idea with the discussion of four of Poe's tales. The purpose is to establish the pattern Poe set as the viable one: the creation of the private detective, the creation of a sidekick character in charge of narrating the facts, and the definition of the method of investigation that keeps on being the basis of detective fiction. A few comparisons with Hammett and Chandler help to define Poe's way of looking at detective fiction.

KEY WORDS: crime, private detective, investigation, narrator.

Don Alfonso Reyes leía, es de imaginar que ensimismado. Era de mañana y se le acercó un famoso psiquiatra mexicano, quien, tras observarlo, le preguntó: “¿También usted lee esas cosas?" (Reyes: 457), pregunta donde es obvio un reproche. ¿Qué literatura fue merecedora de tal desdén? Si les da por suponer que la policiaca, habrán acertado sin del todo conseguirlo. Y digo esto porque Reyes se entretenía con una novela policial, término que prefería al otro o al de detectivesca sin explicar por qué. ¿Hay algo de novedoso en esto? Sí, el enteramos de que don Alfonso gustaba del género, pues el agregado, el desprecio que se adivina en las palabras del psiquiatra, viene de antiguo y estuvo muy extendido. $\mathrm{Y}$ digo estuvo porque este tipo de narrativa se ha ido ganando el respeto de la crítica.

Una de las bases de tal desconsideración es suponer a la literatura policiaca o detectivesca un mero pasatiempo sin bondades estéticas. W. H. Auden, que la leía gozoso, así lo confiesa: la encuentra mera lectura para matar el tiempo. Y si al sumergirse en 
una novela policiaca descubría ya conocerla, no la terminaba. ¿Para qué, si estaba al tanto de la identidad del culpable? Entonces, es de preguntarse si la buena recepción mercantil del género está en función directa de su banalidad. Creo prudente atender lo que al respecto dice uno de los más destacados escritores del género negro: Raymond Chandler. En su ensayo "El sencillo arte de asesinar" asegura, con plena razón, que no hay malos géneros, sino malos escritores. Agrega entonces los rasgos característicos de la novela de misterio y concluye: "Nada queda por examinar, excepto el decidir si está lo bastante bien escrita como para ser una buena narrativa" (Chandler: 977). Es decir, cada novela y cada cuento policiaco determinan su valía mediante la calidad de sus constituyentes literarios.

Volvamos a Auden. Tras asegurar que "las narraciones policiacas nada tienen que ver con las obras de arte" (Auden: 301), aunque aceptando que es una opinión muy personal, las define como obligadas a seguir una fórmula e, incluso, precisa cuál es dicha fórmula. Hela aquí: "ocurre un asesinato: hay muchos sospechosos; se van eliminando a todos los sospechosos, menos a uno, quien es el asesino; se lo arresta o muere" (ibid.: 301-302). Es de reconocer que lo descrito funciona bien para un grueso de las novelas llamadas en inglés "Whodunit" y en español "de enigma", pero no para todo lo escrito en el campo de lo policiaco. El propio Alfonso Reyes aceptaba la presencia de una fórmula como debilidad del género, pero también aceptaba que hacia 1925 se dio un cambio hacia lo que él llamó un tipo de novela "específicamente contemporáneo". Justo aquel que Chandler, entre otros muchos, representaba.

Pero la variedad llamada "de enigma" continúa muy viva, si bien modificada un tanto por contagios con la serie negra. Agatha Christie fue una firme seguidora de la novela "de enigma". P. D. James, que mucho la supera en calidad literaria, continúa la tradición, pero habiéndola modernizado. Ahora bien, no existe género sin antecedentes y sin un punto de origen. Reyes no titubea en cuanto a los que él llama los precursores definidos de este tipo de literatura: Poe, Collins, Conan Doyle. Puede recurrirse a otras opiniones: no desmienten a don Alfonso, bien que puedan agregar al trío algunos precursores, como es el caso del inspector Eugène-François Vidocq (1775-1857), hombre con graves antecedentes criminales que, paradoja, creó los cuerpos policiacos franceses, la Sûreté Nationale, y publicó una autobiografía que le escribieron. Se rumora que Victor Hugo lo tomó de modelo para su villano inspector de Los miserables. Se rumora que Poe lo tomó de modelo para su Augusto Dupin.

No hay historia de la narrativa policiaca que pueda evitar la mención de Edgar Allan Poe (1809-1949), puesto que a él se atribuye el nacimiento del género con la publicación, en 1841, de "Los crímenes de la calle Morgue". Lo que sí cabe preguntarse es ¿por qué justo en ese momento se da el surgimiento del género? Porque asesinatos los hubo desde la Biblia, y jugueteando un tanto con las ideas pudiera decirse que Yavhé fue el primer detective, bien que conociera de antemano lo que iba a suceder. Sin embargo, sólo a principios del siglo XIX se establece dicha narrativa. ¿Por qué? Román Gubern tiene una respuesta convincente: 
No cabe duda de que factores históricos como el desarrollo de las grandes concentraciones urbanas (y el consiguiente auge de la criminalidad), la aparición de las primeras "policías secretas" (Fouché en Francia) y el nacimiento de la prensa sensacionalista, llamada luego "prensa amarilla" en los Estados Unidos [... ] fueron elementos decisivos, y escasamente estudiados, en la aparición histórica de este género, que nace oficialmente con "Los crímenes de la calle Morgue", de Poe... (Gubern: 10).

Con lo cual llegamos a Poe. Ninguna historia de la literatura estadounidense puede omitirlo sin quedar trunca, pero en varias ocasiones los especialistas han disminuido la importancia de su presencia. Se lo considera una especie de flor exótica en el jardín de la literatura estadounidense, pues rara vez, si alguna, reprodujo en sus textos el sabor local. O si se lo quiere expresado en palabras de un crítico: "Un hecho sorprendente en la obra de Poe son sus escasas referencias a datos y acontecimientos de su lugar y época" (Abel: 224), siendo que lo contrario ocurría con los autores que le eran contemporáneos. Por un tiempo casi olvidado, regresó a la atención pública gracias al impulso que le dio Charles Baudelaire al traducirlo al francés, en opinión de Hervey Allen porque en ocasiones "se piensa que las traducciones llegaron a superar a los originales" (Allen: VII), opinión que considero injusta. Pero sí es de subrayar que el poeta francés respetaba mucho las teorías literarias de Poe, que lo influyeron, y le dio impulso.

Sin embargo, aquí nos interesa el Poe narrador. Si hago caso a la edición que tengo de su narrativa y sus poemas, la cual presume de completa, el autor escribió setenta y dos cuentos y una novela: La narración de A. Gordon Pym, mejor conocida en español como Las aventuras de Arturo Gordon Pym, puesta ante los lectores en 1838. A Poe se lo considera un representante del movimiento romántico, así como un frecuentador de la literatura gótica, un impulsor de la de ciencia ficción y el creador de la policiaca, que él llamaba de "raciocinio" para oponerla a aquellos otros textos donde se imponían las tendencias emocionales. Su primer libro fue de poesía. Titulado Tamerlain y otros poemas, apareció en forma anónima, pues la portada se limitaba a informar que "un bostoniano" era el autor. Ocurrió esto en 1827. El primer libro de cuentos pertenece a 1840. Incluía el nombre del autor y se llamaba Cuentos de lo grotesco y lo arabesco, título revelador de lo que Poe pensaba de sus creaciones.

Respecto a los policiacos, cuatro piezas están reconocidas como plenamente del género y a la quinta se la piensa con rasgos de lo detectivesco. Esto último se refiere a "El escarabajo de oro", que no formará parte de mis intereses porque lo considero perteneciente al campo de los criptogramas y no de la investigación policiaca, en la cual inserto sin titubeos a los cuatro restantes. Que son: "Los crímenes de la calle Morgue”, de 1841, aparecido por vez primera en Graham's Magazine. A 1842 pertenece "El misterio de Marie Rogêt", publicado en los números de noviembre y diciembre de Ladies' Companion. En la revista The Gift tuvo acogida "La carta robada", del año 1844, y en ese mismo año Poe escribió “"Tú eres el hombre”".

Llamar "de enigma" a una modalidad de la narrativa policiaca y llamar "género negro" a otra significa establecer una diferencia, en este caso profunda. La preciso, 
aunque muy reducida en sus términos: la narrativa de enigma se centra en el desentrañamiento de un misterio, generalmente un asesinato, poniéndose en segundo término otras consideraciones, como pudieran ser el trasfondo ético, la psicología de los personajes o cuestiones de índole social. La novela negra, por el contrario, toma como excusa el enigma para hacer una crítica acre de una cierta sociedad, crítica que no desprecia entrar en consideraciones de orden moral. Con base en esto, ningún problema hay en asegurar que Poe funda la literatura de enigma. La época no estaba madura aún para lanzarse a explorar las otras propuestas, más cuestionadoras del entorno. En cuanto a consideraciones éticas, la literatura de enigma propone que la sociedad, en términos generales, está sana excepto por las pústulas representadas por los criminales. La literatura negra describe a la sociedad como enferma, siendo expresión palpable de esa condición quienes asesinan.

En tres de sus cuentos Poe elige como topos de lo narrado la ciudad de París, siendo una posibilidad de explicación que la encontrara más desarrollada, si cabe el término, como marco de contención de lo relatado. O dicho en palabras del narrador de "Marie Rogêt", está "la enorme frecuencia, en las grandes ciudades, de atrocidades tales como la descrita" (Poe: 172). Una razón adicional la hemos sugerido anteriormente: el autor tiende a eximirse de situar poemas y cuentos en Estados Unidos. Aunque ocurrida en el inexistente condado de Rattleborough, la cuarta narración tiene un suficiente sabor norteamericano. En cuanto a época, los narradores limitan la información a escribir $18 \ldots$, lo cual permite precisar sin precisar y que fue, por otro lado, un recurso frecuentado por la literatura de la época.

Ernst Mandel asegura que "el tema real de los primeros relatos policiacos no es, entonces, el crimen o el asesinato, sino el enigma en sí. El problema es analítico, no social ni jurídico" (29), descripción exacta de lo que hace Edgar Allan Poe. Pero aquí es necesaria una salvedad: el más insistente de los cuentos, respecto al análisis, es el primero de ellos, "Los crímenes de la calle Morgue", que inicia con una meditación bastante larga sobre el proceso investigativo, meditación de tendencias filosóficas y ensayísticas. Sirva esta cita de ejemplo: "Entre el ingenio y la habilidad analítica se da una diferencia mucho mayor, de hecho, que entre la fantasía y la imaginación, pero de carácter muy estrictamente análogo. De hecho, se descubrirá que lo ingenioso es siempre fantasioso, y lo verdaderamente imaginativo jamás otra cosa que analítico" (ibid.: 143). Tal dice el protagonista del cuento, que más adelante utilizará los crímenes del título para sujetar a prueba lo antes afirmado. De las veintiocho nutridas páginas que el texto presenta, dos y media están dedicadas a crear este marco filosófico referencial, seis a los datos sobre el crimen presentados por la prensa y el resto a la investigación llevada a cabo por C. Auguste Dupin.

La entrada en materia es mucho más expedita en "El misterio de Marie Rogêt" y prácticamente in medias res en la "Carta robada", con presentación muy rápida de los acontecimientos en "Tú eres el hombre". Se diría entonces que, tras establecer en su primera incursión en el género lo que para él significaba cuento de "raciocinio", Poe obedeció después los mandatos de lo meramente narrativo, aunque parte de la 
crítica se queja de "Marie Rogêt", pues lo considera casi un ensayo por su falta de ligereza en hacer avanzar la trama. Algo hay de razón en lo dicho. Cuando se llega a las aventuras del Padre Brown se confirma de inmediato que G. K. Chesterton (18731936) había aprendido la lección, pues informa narrando. Cabe preguntarse ¿sabía Poe que inauguraba un género y quiso asentar sus cimientos? Lo haya considerado o no así, lo cierto es que estableció ciertos elementos que no han dejado de aparecer en la literatura del género.

Por ejemplo, el detective aficionado C. Auguste Dupin pone en marcha una abundante línea de personajes que se atienen a los rasgos principales de aquél creado por Poe, el de ser investigadores privados, si bien una de estas características parece sólo convenirle a él: su afán de resolver enigmas por el simple afán de resolverlos, por el goce que le da comprobar que el intelecto vence siempre. Porque, proviniendo de una familia ilustre, según adjetivo del narrador, Dupin vive un tanto en la penuria mas sin ocurrírsele que el trabajo de detective pudiera redituarle ganancias. Queda claro que el desciframiento de enigmas es para él un mero juego intelectual. También lo es para Sherlock Holmes (con una primera aparición en 1882), quien mediante su dedicación a tareas detectivescas elimina el aburrimiento de la vida, aburrimiento que, no habiendo un caso a la vista, resuelve consumiendo opio o tocando el violín. Al padre Brown (que se inaugura como personaje en 1911) la tarea de investigador le sirve para explorar, aparte de los crímenes, cómo funciona el bien y el mal que existe en los hombres. Posteriormente se dará un detective que de aficionado pasará a profesional, del cual es ejemplo contundente el Nero Wolfe de Rex Stout (1882-1975), cuyo debut ocurre en 1934.

Así pues, C. Auguste Dupin inaugura un modo de ser detective. Sir Arthur Conan Doyle (1859-1930) confiesa haber tomado de él algunos rasgos para conformar a su famoso investigador, bien que otros los derivara del doctor Joseph Bell, con quien Doyle trabajó por un tiempo. Sucede, sin embargo, que la obsesión por vencer enigmas mediante el raciocinio pertenece a Dupin, pues el anónimo narrador de " Thou Are the Man"” se pone en otra tesitura: aquélla de restablecer en el mundo un orden ético, lo cual lo aproxima (sin excesos) a la serie negra. En efecto, el anónimo narrador sospecha que el personaje llamado (irónicamente) Charles Goodfellow es el verdadero culpable del asesinato que se investiga y bajo mano, sin nada informarle al lector en la superficie, va confirmando sus deducciones. Una vez confirmadas éstas, prepara una trampa en la que, con plena obediencia, cae el verdadero culpable. Así se establece una diferencia narrativa muy considerable entre los tres cuentos de Dupin y el de narrador anónimo.

Como ya dije anteriormente, el primer cuento establece un marco de referencia conceptual, pasa a informar de los datos que se conocen sobre el crimen y procede a la investigación. Con bastante menos marco referencial, los dos siguientes relatos con Dupin de personaje central siguen el mismo orden. Compárese con el inicio de "Tú eres el hombre": "Ahora interpretaré el papel de Edipo ante el enigma de Rattleborough. Expondré ante ustedes - como sólo yo puedo hacerlo- el secreto de la trama que permitió el milagro de Rattleborough" (Poe: 490). Nótese cómo el narrador de 
inmediato nos hace insinuaciones de cuál es su cultura, cuál es la importancia de su presencia y de que se dio en Rattleborough un milagro cuya naturaleza irá dejando al descubierto el lento andar de la historia. El narrador es muy ducho, ya que sin subrayarlo va poniendo ante la percepción del lector una serie de sutilezas que, examinadas en retrospectiva, nos dejan ver su importancia. Conviene dar un ejemplo. ¿La causa del crimen? Que el sobrino del muerto le dio un mal golpe al verdadero culpable. Éste, para vengarse, hace que el asesinato parezca un acto del sobrino para quedarse con el dinero del tío. ¿Por qué entra en sospechas el narrador? Porque "el exceso de franqueza del Sr. Goodfellow me había disgustado, así como provocado en mí sospechas desde el principio" (Poe: 500). Por tanto, interviene calladamente para que se restablezca la justicia y, claro, el orden social.

Los tres cuentos anteriores son narrados por un testigo presencial, que lo es porque acompaña a Dupin en sus indagaciones. Con la creación de tal personaje Poe introduce en la literatura de enigma un rasgo que permanecerá en ella por algún tiempo: el del auxiliar del investigador, que tiene la misma categoría social de éste en los casos de Poe y Doyle, para transformarse en un empleado a sueldo en la narrativa de Stout, sin que ello disminuya la importancia del sujeto. En la mayoría de la literatura posterior se recurrirá a un narrador heterodiegético y, en la serie negra, por lo común se insistirá en la primera persona. ¿Por qué elige Poe este tipo de acompañante? Seguramente por el enfoque dado: el narrador establecerá los hechos desde su perspectiva, que estará limitada a lo que presencia. Sirve de vía de transmisión de lo que piensa el detective, con la obligación de explicar cómo se llegó a esas deducciones. Pero sobre todo sirve para que el investigador tenga una excusa que le permita detallar el proceso de revelación de la verdad. Para ello se necesita que la capacidad deductiva del narrador sea menor que la del protagonista, puesto que así se explica la necesidad de que éste le ponga en claro, con toda la minuciosidad requerida, cómo llegó a las conclusiones alcanzadas.

En el caso de Dupin, el narrador testigo tiene el cuidado, y la delicadeza, de ponernos al tanto de todo lo que es pertinente: cómo se conocieron, por qué terminan viviendo juntos, cuáles son los hábitos del investigador y cuáles sus preferencias. Digamos, "era una rareza en los gustos de mi amigo (¿pues de qué otra manera llamarla? ) estar enamorado de la noche porque sí" y viene la confesión de que quien narra cedió "a esos extravagantes caprichos con total abandono" (ibid.: 144), mediante lo cual establece su papel de subordinado, una subordinación que, lo he dicho ya, no lo rebaja como individuo. ¿Nos extrañará que un año después participe en la investigación del misterio de Marie Rogêt? ¿O que "varios años después" participe en el caso de la carta robada? El inicio de este cuento nos aclara la vida que llevan entre un caso y otro: "En París, poco después de haber anochecido una tormentosa tarde en el verano de 18 _ gozaba yo el doble placer de la meditación y de una pipa de espuma de mar en compañía de mi amigo C. Auguste Dupin” (ibid.: 208). De haber alargado Poe la presencia de lo detectivesco en su cuentística, esos intermedios de paz entre un caso y otro habrían funcionado como aquellos similares en la narrativa policiaca de Doyle. 
Es el momento de preguntarse ¿y qué de la policía? Pues existe y funciona en los cuentos de Dupin como personaje, pero de un modo que le conviene a las intenciones de la narración. La principal de éstas consiste en aclarar que hay otros tipos de investigación, muy distintos al empleado por el detective, que son lógicos pero ineficaces e ineficaces por atenerse a una lógica superficial, incapaz de desentrañar las verdaderas razones del crimen ocurrido. En ocasiones, el inspector de policía merece casi el adjetivo de tonto. Según avanzamos en el tiempo, esta figura pierde su aire caricaturesco y gana el papel de protagonista, de lo cual son prueba sólida el inspector Adam Dalgliesh, creado por P. D. James, y el comisario Guido Brunetti, ideado por Donna Leon. Pero cuando Poe escribe el sistema policiaco era relativamente joven y carecía de los apoyos científicos que hoy son la rutina en cualquier serie televisiva policiaca. De ahí la importancia de la observación personal.

Una primera clave para diferenciar los dos métodos de investigación aparece muy temprano en "Los crímenes de la calle Morgue". Hela aquí: "El conocimiento necesario consiste en saber qué observar" (ibid.: 142). Es decir, puestos en la escena del crimen, percibir cuáles son los indicios importantes, los que verdaderamente dan la información que permitirá resolver el enigma, capacidad de hacer observaciones que en Dupin provoca una excitación mental deleitosa. En cambio, la policía no sale de sus rutinas, de buscar lo obvio, sistema que aplica en un caso tras otro. ¿A quién pone como ejemplo de esto Dupin y por lo tanto Poe? Al antes mencionado Eugene Vidocq. Se le reconocen méritos, desde luego, pero también desméritos, uno de los principales el siguiente: "Menoscaba su visión acercando demasiado el objeto" (ibid.: 152), lo cual le permite apreciar los detalles del mismo mas no su totalidad. Y la solución del enigma está en que la suma de los detalles permita comprender el todo, no importando que el todo apunte a una solución al parecer inaceptable, como termina sucediendo en este cuento, donde un orangután es el causante de las muertes ocurridas.

¿Qué se deriva de lo anterior? Que la prefectura desee la participación de Dupin en varios casos, muchos de los cuales el investigador rechaza por no encontrarlos interesantes o, al menos, lo bastante atractivos como para sacarlo de sus "ensoñaciones taciturnas", según las describe el narrador. Hasta que aparece "El misterio de Marie Rogêt" y más tarde "La carta robada", cuento al principio del cual se deja caer en casa de Dupin "nuestro viejo conocido el señor G , prefecto de la policía parisiense" (ibid.: 208) para ofrecerle un enigma capaz de estimularle la curiosidad. Los detalles que he venido comentando son aspectos que se repetirán, con ciertas modificaciones no sólo pertinentes sino necesarias, en los cuentos de Doyle: los intermedios de aburrimiento entre caso y caso, aliviados, como ya informé, con tocar el violín o consumir opio; la amistad Holmes-Watson; la capacidad de análisis del investigador privado y su al parecer inacabable fondo de conocimientos variados, y el papel asignado a los representantes de la ley. Pero incluso llega más allá en el tiempo: el Nero Wolfe de Rex Scout halla en el cultivo de orquídeas y en la gastronomía bálsamos para el aburrimiento que lo consume entre una investigación y otra, dándose también, como ya dijimos, la presencia de Archie Goodwin, su ayudante y, como sucede en los tres casos mencionados, autor de los relatos que leemos. 
Vemos así cómo Poe asienta desde un principio varios rasgos que permanecerán firmes en la literatura de enigma: la presencia de un misterio duro de resolver como reto a la inteligencia del investigador aficionado, la presencia de éste como una figura singular por sus características personales, el interés por el enigma como enigma y no por razones de orden ético o moral (como sí se dan en el padre Brown), la presencia de un compañero de aventuras y la estructura del relato. Es decir, la presentación del caso, la acumulación de datos, y la revelación final del misterio, con las explicaciones pertinentes de cómo se la dedujo. Es un patrón al que obedecerá en distintos grados la literatura posterior.

Sin embargo, no pienso que terminen allí las aportaciones del autor. Por ejemplo, vuelvo a "Los crímenes de la calle Morgue" y he de reconocer que inaugura una línea de misterios fáciles de resumir: aquellos sucedidos en una habitación cerrada por dentro, lo cual plantea la siguiente cuestión: ¿por dónde y de qué manera entró o por dónde y de qué manera salió el asesino? No hay duda de que el texto más conocido en esta variante policiaca es El misterio del cuarto amarillo, publicado en 1907 por Gaston Leroux (1868-1927), pero el cuento de Poe es el que inaugura la serie. Por otro lado, con "La carta robada" inicia otra modalidad policiaca: aquella en la cual no se descubre la verdad simplemente porque está a la vista de todos, como sucede con la carta robada. Un ejemplo posterior de lo mismo aparece en el cuento de Chesterton llamado, justamente, "El hombre invisible", donde el culpable se disfraza de cartero y nadie, por lo mismo, le presta atención. Como le dice el padre Brown al personaje llamado Angus: "No está usted loco... simplemente es poco observador" y explica que nadie se fija en lo cotidiano justamente por cotidiano y, agregaría Poe, también se rechaza todo lo que parece incongruente, con lo cual tenemos los dos extremos de un continuo dentro de lo policiaco.

Ahora bien, ¿qué propone "El misterio de Marie Rogêt"? No es de afirmar que introduce el aprovechamiento de un hecho real para transformarlo en narrativa, puesto que desde siempre ha sido uno de los sustentos fundamentales de la creación literaria. Tampoco el hacer narrativa periodística a lo Truman Capote, puesto que se han cambiado los nombres de los personajes y el de la localidad donde ocurrieron los hechos. Pudiera tratarse de un intento de ocultar, por razones de delicadeza o cortesía, la identidad real de los participantes en el caso. Pero las notas aclaratorias que acompañan al cuento parecen negar esta posibilidad. Por otro lado, la prensa de entonces atendió de modo obsesivo al asesinato de Mary Rogers, para mencionar ya el nombre real de la víctima, cayendo incluso en el amarillismo. Por tanto, el disfraz puesto por el autor a los personajes de su cuento resultaba transparente para un lector de la época. En busca de razones, cito del propio texto de Poe: "Cuando la publicación original de 'Marie Rogêt', se consideraron innecesarias las notas que hoy se agregan; pero el transcurso de varios años desde ocurrida la tragedia en que el cuento se basa hace obligatorio el ofrecerlas" (ibid.: 169), sumándosele además datos sobre el acontecimiento real.

Tal vez la razón principal que llevó a transformar el suceso en cuento se dé unas líneas después: "el misterio que acompañaba [la tragedia] seguía sin resolverse en el momento de escribirse y publicarse este texto (noviembre de 1842)" (idem.), pues se 
agrega que el autor dedujo cómo y por quién podría haberse cometido el asesinato. La transparencia del nombre elegido (Marie Rogêt) era un guiño de complicidad al lector enterado. Es decir, en ese noviembre de 1842 el cuento establecía en sus primeras páginas una descripción del crimen atenida a lo ocurrido, incluyendo bajo disfraz notas tomadas de la prensa de aquel entonces. Establecidos los hechos, el resto de la narración derivaba de las especulaciones y deducciones a las que sin mayor prisa llegaba C. Auguste Dupin. Tengo con ello que volver a un punto ya examinado: lo importante para el autor, y por tanto para Dupin, era el proceso de investigación, puntillosamente descrito en el cuento, y no consideraciones éticas o morales que, de presentarse, lo hacen como meros soportes del interés central: la solución del delito.

Aquí es de mencionar un aspecto interesante: en el caso real el examen de los hechos se llevó a cabo lejos de donde éstos ocurrieron, no así en la narración. Por otro lado, en ésta Dupin entra en acción a un mes de haberse cometido el asesinato, situación harto desusada en las muestras del género. Es decir, el investigador trabaja con base en datos indirectos, no a partir de una observación personal de los lugares donde se dio el hecho. Pero si se considera que justo eso se adapta mejor a los gustos del protagonista, la situación resulta ideal. Es de comentar que Dupin entra así de tarde en la investigación porque lo invita el prefecto de policía, sin duda ya preocupado por la lentitud con que marcha la investigación. Preciso como en el cuento precedente (no se olvide que "Marie Rogêt" aparece con el subtítulo de "Una secuela de 'Los crímenes de la calle Morgue"”), el narrador detalla sin cansancio, y tal vez en demasía, el proceso de aclaración del crimen que, desde luego, quedará muy distanciado de lo supuesto por la policía. Tal vez porque, son palabras de Dupin, "no es el menor de los errores en investigaciones de este tipo el limitar las pesquisas a lo inmediato, con total desprecio de los sucesos colaterales o circunstanciales" (ibid.: 191), con lo cual, a lo largo de ios tres cuentos protagonizados por Dupin, se establecen sus creencias y hábitos de investigador, muchos de los cuales pasarán como herencia a futuros investigadores.

El crimen queda resuelto, como es lo obligatorio en el género. Finaliza el texto con el narrador señalando lo que él llama "coincidencias" entre el caso de Mary Cecilia Rogers y el de Marie Rogêt. ¿Coincidencias?, se pregunta un lector inteligente. Al parecer, estimulado por el hecho ocurrido en el mundo concreto, Poe se lanzó a escribir el cuento buscándole solución antes de que el suceso real la tuviera. La capacidad deductiva del autor quedó comprobada al verse más tarde que lo que se sabía del caso verdadero coincidía en mucho con lo propuesto por el autor como explicación. No debe extrañar, pues Thomas Narcejac nos pone al tanto de lo siguiente: "En 1841 apareció una novela de Dickens, Barnaby Rudge, que contenía el relato de un crimen misterioso. Edgar Poe, después de haber leído las primeras páginas de la obra, descubrió el enigma; y reflexionando sobre el método analítico que había seguido, descubrió al mismo tiempo las reglas de la investigación policiaca y las del relato policiaco" (Gubern: 54). He aquí pues a Dickens como promotor indirecto del relato policial.

Otro aspecto interesante de "Marie Rogêt", el cual sólo mencionaré de pasada, son los vasos comunicantes que establece entre realidad y narrativa, terreno siempre 
interesante de explorar. Con base en un estudio comparativo, se terminará precisando qué del hecho real tomó Poe como material constructivo de su cuento, qué dejó fuera y, quizá de importancia especial, cómo transformó aquellos aspectos del caso utilizados como elementos narrativos. Pero lo que importaba a estas consideraciones está dicho: Poe es el fundador innegable de la literatura policial de enigma. Lo consigue mediante una escasa producción de cuatro relatos, tal vez cinco si alguien insiste en aceptar "El escarabajo de oro" como policial, en tres de los cuales establece la presencia de lo que considero el primer investigador privado: C. Auguste Dupin, y proponiendo una estructura narrativa que perdurará en el género. Por otro lado, crea la figura del acompañante del investigador, que se encargará de narramos los hechos, y la figura del inspector de policía prisionero de los métodos de averiguación convencionales, que le impiden llegar a la verdad. Todo ello sin olvidar que Poe exploró otras variantes del cuento, quedando como uno de los narradores destacados del siglo XIX.

\section{Obras citadas}

ABEL, Darrel. 1963. American Literature. Volume 2: Literature of the Atlantic Culture. Nueva York: Barrow.

Allen, Hervey. [s.f.]. "Introduction". The Complete Tales and Poems of Edgar Allan Poe. Nueva York: The Modem Library. (A Modem Library Giant, G 40). Pp. VVIII.

Auden, W. H. 1956. "The Guilty Vicarage: Notes on the Detective Story, by an Addict". The Critical Performance. Ed. Stanley Edgar Hyman. Nueva York: Vintage Books. Pp. 301-314.

ChandLer, Raymond. 1995. "The Simple Art of Murder". Later Novels \& Other Writings. Nueva York: The Library of America. Pp. 977-992.

Gubern, Román, ed. 1982. La novela criminal. 2a. ed. Barcelona: Tusquets Editores. (Cuadernos Ínfimos, 10)

Mandel, Ernst. 1986. Crimen delicioso. Trad. Pura LóPez Colomé. México: UNAM, Dirección de Literatura. (Textos de Ciencias Sociales)

PoE, Edgar Allan, [s. f. ]. The Complete Tales \& Poems. Nueva York: The Modern Library. (A Modem Library Giant, G 50)

REYES, Alfonso. 1981. "Sobre la novela policial”. Obras completas, t. IX. México: FCE. (Letras Mexicanas). Pp. 457-461. 